1. Druker, B.J., et al. 2006. Five-year follow-up of patients receiving imatinib for chronic myeloid leukemia. N. Engl. J. Med. 355:2408-2417.

2. Li, J., et al. 2007. TNF-alpha induces leukemic clonal evolution ex vivo in Fanconi anemia group C murine stem cells. J. Clin. Invest. 117:3283-3295.

3. Bagby, G.C., and Meyers, G. 2007. Bone marrow failure as a risk factor for clonal evolution: prospects for leukemia prevention. Hematology. 2007:40-46.

4. Rosenberg, P.S., Greene, M.H., and Alter, B.P. 2003 Cancer incidence in persons with Fanconi anemia. Blood. 101:822-826.

5. Bagby, G.C., and Alter, B.P. 2006. Fanconi anemia. Semin. Hematol. 43:147-156.

6. Haneline, L.S., et al. 1998. Multiple inhibitory cytokines induce deregulated progenitor growth and apoptosis in hematopoietic cells from FAC - / mice. Blood. 91:4092-4098.

7. Lensch, M.W., Rathbun, R.K., Olson, S.B., Jones, G.R., and Bagby, G.C., Jr. 1999. Selective pressure as an essential force in molecular evolution of myeloid leukemic clones: a view from the window of Fanconi anemia. Leukemia. 13:1784-1789.

8. Li, X.X., et al. 2005. Ex vivo culture of Fancc/- stem/ progenitor cells predisposes cells to undergo apop- tosis, and surviving stem/progenitor cells display cytogenetic abnormalities and an increased risk of malignancy. Blood. 105:3465-3471.

9. Mori, H., et al. 2002. Chromosome translocations and covert leukemic clones are generated during normal fetal development. Proc. Natl. Acad. Sci.U. S. A. 99:8242-8247.

10. Liu, F., et al. 2008. Csf $3 r$ mutations in mice confer a strong clonal HSC advantage via activation of Stat5. J. Clin. Invest. 118:946-955.

11. Dale, D.C., et al. 2000. Mutations in the gene encoding neutrophil elastase in congenital and cyclic neutropenia. Blood. 96:2317-2322.

12. Grenda, D.S., et al. 2007. Mutations of the ELA2 gene found in patients with severe congenital neutropenia induce the unfolded protein response and cellular apoptosis. Blood. 110:4179-4187.

13. Kollner, I., et al. 2006. Mutations in neutrophil elastase causing congenital neutropenia lead to cytoplasmic protein accumulation and induction of the unfolded protein response. Blood. 108:493-500.

14. Link, D.C., et al. 2007. Distinct patterns of mutations occurring in de novo AML versus AML arising in the setting of severe congenital neutropenia.
Blood. 110:1648-1655

15. Germeshausen, M., Ballmaier, M., and Welte, K. 2007. Incidence of CSF3R mutations in severe congenital neutropenia and relevance for leukemogenesis: Results of a long-term survey. Blood. 109:93-99.

16. Hunter, M.G., and Avalos, B.R. 2000. Granulocyte colony-stimulating factor receptor mutations in severe congenital neutropenia transforming to acute myelogenous leukemia confer resistance to apoptosis and enhance cell survival. Blood. 95:2132-2137.

17. Lo Ten Foe, J.R., et al. 1997. Somatic mosaicism in Fanconi anemia: Molecular basis and clinical significance. Eur. J. Hum. Genet. 5:137-148.

18. Mankad, A., et al. 2006. Natural gene therapy in monozygotic twins with Fanconi anemia. Blood. 107:3084-3090.

19. Boyle, K., et al. 2007. The SOCS box of suppressor of cytokine signaling-3 contributes to the control of G-CSF responsiveness in vivo. Blood. 110:1466-1474.

20. Alter, B.P. 2007. Diagnosis, genetics, and management of inherited bone marrow failure syndromes. Hematology Am. Soc. Hematol. Educ. Program. 2007:29-39.

\title{
Currying favor for the heart
}

\author{
Jonathan A. Epstein \\ Department of Cell and Developmental Biology, Cardiovascular Institute, and Institute for Regenerative Medicine, \\ University of Pennsylvania, Philadelphia, Pennsylvania, USA.
}

\begin{abstract}
Curcumin, a commonly available spice and alternative medicine, has been tested in the laboratory and the clinic for activity against a wide range of diseases. It is thought to possess antiinflammatory and antioxidant activities and may also function to inhibit histone acetyl transferases, which activate gene expression via chromatin remodeling. Two reports in this issue of the JCI, by Morimoto et al. and $\mathrm{Li}$ et al., suggest that curcumin may inhibit cardiac hypertrophy in rodent models and provide beneficial effects after myocardial infarction or in the setting of hypertension (see the related articles beginning on pages 868 and 879, respectively). These results will spur further mechanistic inquiry into the role of chromatin remodeling in the regulation of cardiac homeostasis.
\end{abstract}

Epigenetics is a term used to describe features of DNA packaging and assembly that modify cellular process and are stably maintained when cells divide, but do not involve changes in DNA sequence. DNA is maintained within the nucleus in an ordered and dynamic structure in association with other proteins, including histones. The complex of DNA and associated proteins is known as chromatin, and an exciting area of active research involves the epigenetic regulation of chro-

Nonstandard abbreviations used: HAT, histone acety transferase; HDAC, histone deacetylase; MEF, myocyte enhancer factor.

Conflict of interest: The author has declared that no conflict of interest exists.

Citation for this article: J. Clin. Invest. 118:850-852 (2008). doi:10.1172/JCI34650. matin structure by enzymes that modify histones. Among these enzymes are those that add or remove acetyl groups on lysine tails of histones. Enzymes that remove acetyl groups are called histone deacetylases (HDACs), and those reactions are reversed by histone acetyl transferases (HATs), which include p300 and CREB-binding protein (CBP). In general, HDACs act as transcriptional repressors, since removal of acetyl groups allows the chromatin to pack more tightly, and access of transcription factors to promoters is restricted. Conversely, HATs tend to function as activators of gene expression.

HDACs have recently been implicated as important regulators of cardiac homeostasis (1). There are at least 11 mammalian HDACs that compose the so-called class
1 and class 2 families, in addition to more distantly related families (2). Inactivation of some of the class 2 HDACs in mice results in cardiac hypertrophy and subsequent heart failure $(1,3,4)$. On the other hand, inactivation of a class 1 HDAC, HDAC2, results in resistance to cardiac hypertrophy (5), which suggests that class 1 and class 2 HDACs may play opposing roles (6). Interestingly, chemical HDAC inhibitors, which block both classes, tend to block hypertrophic responses (6-8). In this issue of the JCI, 2 papers examine the effects of curcumin on the heart and conclude that this commonly available spice blocks HAT activity and prevents cardiac hypertrophy and failure in rodent models $(9,10)$.

\section{Curcumin and cardiac hypertrophy}

Curcumin is a polyphenol responsible for the yellow color of the curry spice turmeric. It has relatively poor bioavailability when taken orally, but also low toxicity (11). It has been touted to possess a myriad of beneficial activities, and clinical trials have been conducted in patients with cancer, rheumatoid arthritis, cystic fibrosis, inflammatory bowel disease, psoriasis, pancreatitis, and other disorders (11-13). Limited data suggest that it possesses antitumor, antioxidant, and antiinflammatory activities. 


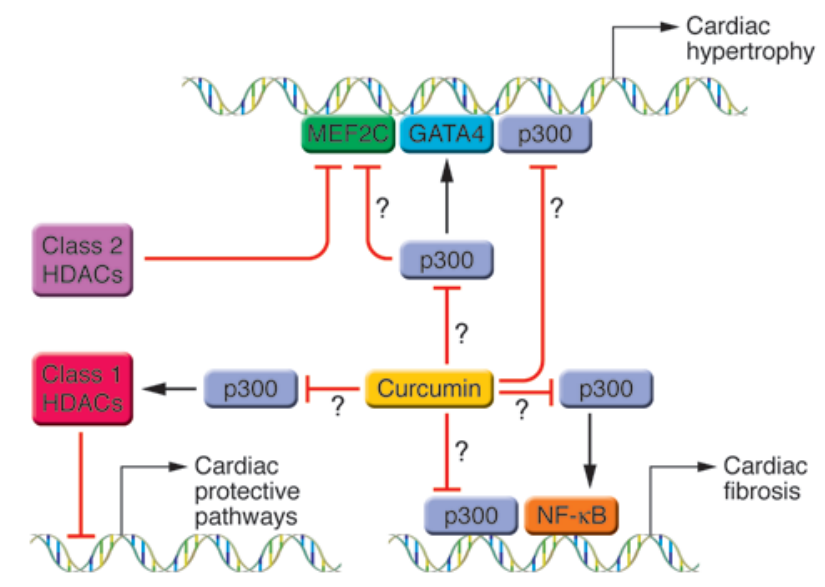

Figure 1

Curcumin can block cardiac hypertrophy in isolated cells and animal models, but the mechanism of action is unclear. This model depicts possible actions (indicated by question marks) of curcumin, including direct inhibition of p300-HAT activity and inhibition of p300 acetylation of GATA4, MEF2C, or NF-кB. p300 functions with GATA4 and MEF2C to activate hypertrophic pathways and may function with NF- $\mathrm{KB}$ to drive pathways important in cardiac fibrosis. Class 2 HDACs interfere with the action of MEF2C and oppose cardiac hypertrophy, while class 1 HDACs have been postulated to inhibit antihypertrophic or protective pathways. Curcumin could potentially inhibit the ability of p300 or other factors to activate class $1 \mathrm{HDACs}$. In this issue of the $\mathrm{JCl}$, Li et al. (9) and Morimoto et al. (10) demonstrate that curcumin can block cardiac hypertrophy in rodent models. Correlative data suggests that possible mechanisms of action may include inhibition of p300-HAT activity and/or inhibition of GATA4 acetylation with subsequent alterations in GATA4 activity and recruitment of p300.

At the molecular level, it has been shown to interfere with the activities of NF- $\mathrm{BB}$, cytochrome $\mathrm{P} 450$, and HIV reverse transcriptase as well as $\beta$ amyloid accumulation, production of inflammatory cytokines, and the activity of p300 (14-16). Nevertheless, few placebo-controlled double-blinded studies have provided convincing evidence for clinical utility (11).

In their study in this issue, $\mathrm{Li}$ et al. (9) now show that rodents treated with oral curcumin are markedly resistant to cardiac hypertrophy produced by banding of the aorta, which mimics the type of cardiac enlargement seen in patients with high blood pressure. Even when treatment was begun 2 weeks after the induction of pressure overload, curcumin was beneficial, and the transition to heart failure was reduced. Treatment correlated with a reduction in histone acetylation and p300-HAT activity. Because p300 has already been implicated in the regulation of cardiac myocyte growth (17-20), the ability of curcumin to block p300-HAT activity in this animal model of hypertrophy suggests a possible mechanism of action. Interestingly, cardiac inflammation and fibrosis were also reduced, perhaps because of inhibition of NF- $\mathrm{KB}$ activity. Although p300 is generally thought to function by modifying chromatin and histone acetylation, it can also function by directly acetylating other proteins. In the heart, p300 can acetylate GATA4 $(20,21)$, a transcription factor strongly associated with hypertrophic gene expression. Curcumin blocked acetylation of GATA4, thus presumably affecting transcriptional activity. Interestingly, myocyte enhancer factor $2 \mathrm{C}$ (MEF2C), which interacts with - and is inhibited by - class 2 HDACs, can also be acetylated (22), although MEF2C acetylation was not studied by these authors. Hence, despite significant correlative data, it remains unclear exactly how curcumin functions to modulate the response of the heart to the stress of increased pressure. More work will be required to determine whether changes in chromatin structure and histone acetylation are important or whether direct regulation of GATA4 or NF- $\mathrm{KB}$, or of other pathways, is responsible.

In a related study in this issue, Morimoto et al. (10) examined different rodent models of heart failure and came to nearly identical conclusions. They studied the effects of oral curcumin on the progression of cardiac hypertrophy and subsequent decompensated heart failure in salt-sensitive hypertensive Dahl rats. They began treatment at 11 weeks of age, when systolic function is still preserved (i.e., before a notable transition to heart failure). After 7 weeks of treatment, they documented significant and beneficial preservation of systolic function in the active treatment group. They also showed that the acetylation of GATA4 that normally accompanies hypertension was reduced by curcumin. The effects of curcumin were also studied when the compound was given to rats 1 week after myocardial infarction. After 6 weeks of treatment, systolic function was improved in the treated animals, and hypertrophy of the noninfarcted myocardium (which is thought to contribute to adverse remodeling) was reduced.

\section{Translating animal studies to clinical practice calls for caution}

The studies by Li et al. (9) and Morimoto et al. (10) suggest that curcumin may have beneficial effects on the heart in the setting of pressure overload or after myocardial infarction. However, translation of these data to humans is not clear cut, and extrapolation should be attempted with caution. Despite numerous interventions that prove efficacious in rodent models after myocardial infarction, for example, few have proven successful in clinical trials (23). Likewise, prior claims of the beneficial effects of curcumin in other settings, largely advanced on the basis of in vitro and animal studies, have proven disappointing $(11,12)$. The mechanism of action of curcumin in the heart remains unclear. While the correlations regarding p300 activity, GATA4 acetylation, and inflammatory pathways are intriguing, additional studies will be necessary to determine which, if any, of these explanations satisfy tests of necessity and sufficiency in vitro and in vivo. These results may provide more specific therapeutic targets and may provide relevant bioassays with which to monitor clinical trials.

If inhibition of $\mathrm{p} 300-\mathrm{HAT}$ activity proves to be the critical mechanism by which curcumin modulates cardiac responses to stress, still more questions will need to be addressed. How can an inhibitor of HDAC activity (such as trichostatin A; refs. 6-8) and an inhibitor of HAT activity (like curcumin) both block hypertrophic responsiveness, when HDAC and HAT pathways oppose one another? Are the same gene expression changes being affected by apparently opposing chemical inhibitors? Is one class of inhibitor blocking a prohypertrophic pathway, while the other is activating a distinct antihypertrophic or protective pathway (Figure 1)? Given the low incidence of reported side effects in 
animal models and in human trials with HDAC and HAT inhibitors $(2,11,24)$, one wonders whether we truly understand the nature and extent of gene expression controlled by these "global" regulators. Do redundant and feedback mechanisms limit the global effects of these inhibitors? The answers to these questions may provide new therapeutic targets for heart failure and may also refine our understanding of the mechanisms by which chromatin remodeling complexes function inside the cell.

Address correspondence to: Jonathan A. Epstein, Department of Cell and Developmental Biology, Cardiovascular Institute, and Institute for Regenerative Medicine, University of Pennsylvania, 1154 BRB II, 421 Curie Blvd., Philadelphia, Pennsylvania 19104, USA. Phone: (215) 898-8731; Fax: (215) 573-2094; E-mail: epsteinj@mail. med.upenn.edu.

1. Backs, J., and Olson, E.N. 2006. Control of cardiac growth by histone acetylation/deacetylation. Circ. Res. 98:15-24.

2. Kelly, W.K., and Marks, P.A. 2005. Drug insight: Histone deacetylase inhibitors - development of the new targeted anticancer agent suberoylanilide hydroxamic acid. Nat. Clin. Pract. Oncol. 2:150-157.

3. Chang, S., et al. 2004. Histone deacetylases 5 and
9 govern responsiveness of the heart to a subset of stress signals and play redundant roles in heart development. Mol. Cell. Biol. 24:8467-8476.

4. Zhang, C.L., et al. 2002. Class II histone deacetylases act as signal-responsive repressors of cardiac hypertrophy. Cell. 110:479-488.

5. Trivedi, C.M., et al. 2007. Hdac2 regulates the cardiac hypertrophic response by modulating Gsk3 beta activity. Nat. Med. 13:324-331.

6. Kook, H., et al. 2003. Cardiac hypertrophy and histone deacetylase-dependent transcriptional repression mediated by the atypical homeodomain protein Hop. J. Clin. Invest. 112:863-871.

7. Antos, C.L., et al. 2003. Dose-dependent blockade to cardiomyocyte hypertrophy by histone deacetylase inhibitors. J. Biol. Chem. 278:28930-28937.

8. Kee, H.J., et al. 2006. Inhibition of histone deacetylation blocks cardiac hypertrophy induced by angiotensin II infusion and aortic banding. Circulation. 113:51-59.

9. Li, H.-L., et al. 2008. Curcumin prevents and reverses murine cardiac hypertrophy. J. Clin. Invest. 118:879-893.

10. Morimoto, T., et al. 2008. The dietary compound curcumin inhibits p300 histone acetyltransferase activity and prevents heart failure in rats. J. Clin. Invest. 118:868-878.

11. Hsu, C.H., and Cheng, A.L. 2007. Clinical studies with curcumin. Adv. Exp. Med. Biol. 595:471-480.

12. Mall, M., and Kunzelmann, K. 2005. Correction of the CF defect by curcumin: hypes and disappointments. Bioessays. 27:9-13

13. Miriyala, S., Panchatcharam, M., and Rengarajulu, P. 2007. Cardioprotective effects of curcumin. $A d v$. Exp. Med. Biol. 595:359-377.

14. Balasubramanyam, K., et al. 2004. Curcumin, a novel $\mathrm{p} 300 / \mathrm{CREB}$-binding protein-specific inhibitor of acetyltransferase, represses the acetylation of histone/nonhistone proteins and histone acet- yltransferase-dependent chromatin transcription. J. Biol. Chem. 279:51163-51171.

15. Chen, Y., et al. 2007. Curcumin, both histone deacetylase and p300/CBP-specific inhibitor, represses the activity of nuclear factor kappa $B$ and Notch 1 in Raji cells. Basic Clin. Pharmacol. Toxicol. 101:427-433.

16. Marcu, M.G., et al. 2006. Curcumin is an inhibitor of p300 histone acetylatransferase. Med. Chem. 2:169-174.

17. Miyamoto, S., et al. 2006. Histone acetyltransferase activity of p300 is required for the promotion of left ventricular remodeling after myocardial infarction in adult mice in vivo. Circulation. 113:679-690.

18. Yanazume, T., et al. 2003. Cardiac $\mathrm{p} 300$ is involved in myocyte growth with decompensated heart failure. Mol. Cell. Biol. 23:3593-3606.

19. Gusterson, R.J., Jazrawi, E., Adcock, I.M., and Latchman, D.S. 2003. The transcriptional co-activators CREB-binding protein (CBP) and p300 play a critical role in cardiac hypertrophy that is dependent on their histone acetyltransferase activity. J. Biol. Chem. 278:6838-6847.

20. Dai, Y.S., and Markham, B.E. 2001. p300 Functions as a coactivator of transcription factor GATA-4. J. Biol. Chem. 276:37178-37185.

21. Kawamura, T., et al. 2005. Acetylation of GATA-4 is involved in the differentiation of embryonic stem cells into cardiac myocytes. J. Biol. Chem. 280:19682-19688.

22. Gregoire, S., et al. 2007. Histone deacetylase 3 interacts with and deacetylates myocyte enhancer factor 2 . Mol. Cell. Biol. 27:1280-1295.

23. Bolli, R., et al. 2004. Myocardial protection at a crossroads: the need for translation into clinical therapy. Circ. Res. 95:125-134.

24. Dokmanovic, M., Clarke, C., and Marks, P.A. 2007. Histone deacetylase inhibitors: overview and perspectives. Mol. Cancer Res. 5:981-989. 